\title{
Nulling at the Keck interferometer
}

\section{Mark Colavita, Gene Serabyn, Peter L. Wizinowich, Rachel L. Akeson}

M. Mark Colavita, Gene Serabyn, Peter L. Wizinowich, Rachel L. Akeson, "Nulling at the Keck interferometer," Proc. SPIE 6268, Advances in Stellar Interferometry, 626803 (23 June 2006); doi: 10.1117/12.672651

Event: SPIE Astronomical Telescopes + Instrumentation, 2006, Orlando, Florida, United States 


\title{
Nulling at the Keck Interferometer
}

\author{
M. Mark Colavita ${ }^{{ }^{a} \mathrm{a}}$, Gene Serabyn ${ }^{\mathrm{a}}$, Peter L. Wizinowich ${ }^{\mathrm{b}}$, Rachel L. Akeson ${ }^{\mathrm{c}}$ \\ ${ }^{\mathrm{a}} \mathrm{Jet}$ Propulsion Laboratory, California Institute of Technology, \\ 4800 Oak Grove Dr., Pasadena, CA 91109 USA \\ ${ }^{b}$ W. M. Keck Observatory, 65-1120 Mamalahoa Hwy, Kamuela, HI 96743 USA \\ ${ }^{c}$ Michelson Science Center, California Institute of Technology, 100-22, Pasadena, CA 91125 USA
}

\begin{abstract}
The nulling mode of the Keck Interferometer is being commissioned at the Mauna Kea summit. The nuller combines the two Keck telescope apertures in a split-pupil mode to cancel the on-axis starlight and coherently detect the residual signal. The nuller, working at $10 \mathrm{um}$, is tightly integrated with the other interferometer subsystems including the fringe and angle trackers, the delay lines and laser metrology, and the real-time control system. Since first 10 um light in August 2004, the system integration is proceeding with increasing functionality and performance, leading to demonstration of a 100:1 on-sky null in 2005. That level of performance has now been extended to observations with longer coherent integration times. An overview of the overall system is presented, with emphasis on the observing sequence, phasing system, and differences with respect to the $\mathrm{V}^{2}$ system, along with a presentation of some recent engineering data.
\end{abstract}

Keywords: Interferometry, Nulling, Keck

\section{INTRODUCTION}

The Keck Interferometer ${ }^{1,2}$ is a NASA-funded project to combine the light from the two Keck telescopes for a variety of science objectives as part of NASA's Navigator Program. A 2 um visibility amplitude mode ${ }^{3,4}$ was commissioned in 2004, and is available to the entire Keck community through the normal proposal process. A 10 um nulling mode is the current focus of engineering development, to be used for the measurement of zodiacal dust around other stars in support of current and future NASA activities in the area of exoplanets. The nuller beam combiner and camera were installed on the summit of Mauna Kea in summer 2004, with first 10 um measurements later that year. The first null measurements took place in early 2005, with stabilized on-sky raw nulls of 100:1 in mid 2005. The current activities are focused on improving performance both in null depth and sensitivity, increasing functionality and operability in preparation for formal commissioning in 2007 , and conducting a shared-risk science program.

Below, we summarize the nuller system implementation, and the current subsystem and system state. Several references provide additional information on system description, ${ }^{5}$ the nuller back-end implementation, ${ }^{6,7}$ and integration and test. ${ }^{8}$ Additional papers in this conference address control issues, ${ }^{9,10}$ observing scenario, ${ }_{11}^{11}$ atmospheric compensation, ${ }^{12}$ and science observations, ${ }^{13}$ as well as the overall facility ${ }^{14}$ and visibility amplitude mode. ${ }^{15}$

\section{ARCHITECTURE AND OBSERVING STRATEGY}

A four-aperture nuller (Figure 1) is implemented by treating the left and right halves of each Keck telescope as largely separate apertures. A modified Mach-Zehnder nuller working at $10 \mathrm{um}$ is implemented on each of the long $(85 \mathrm{~m})$ apertures to null out the light from the star to improve detectability of surrounding zodiacal dust or other extended emission (there is no fundamental SNR gain as thermal background is dominant in any ground-based MIR implementation). The output of the two nullers - each of which can be considered as a telescope with an unusual beam

*mark.colavita@jpl.nasa.gov

Advances in Stellar Interferometry, edited by John D. Monnier,

Markus Schöller, William C. Danchi, Proc. of SPIE Vol. 6268,

626803, (2006) -0277-786X/06/\$15 - doi: 10.1117/12.672651

Proc. of SPIE Vol. 6268 626803-1 
pattern - is combined in a Michelson interferometer (henceforward, the cross-combiner), with an effective baseline of $\sim 6$ $\mathrm{m}$, to measure the coherent leakage signal.

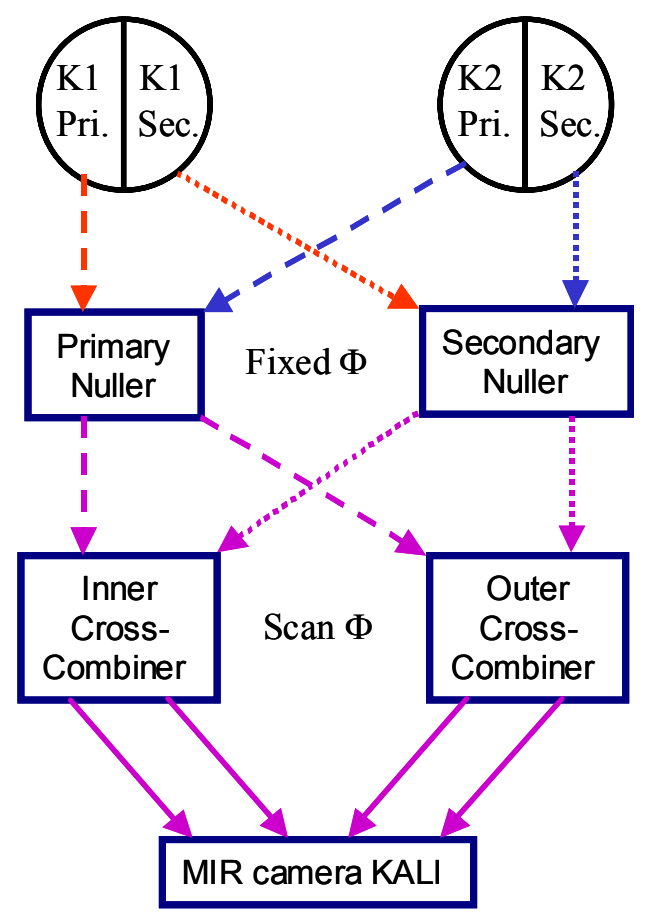

Figure 1. Four-aperture nuller schematic.

\subsection{Observing strategy}

Properly normalized - more below - the null leakage is the science observable, and the observing strategy follows that familiar from $\mathrm{V}^{2}$ observing. Local calibrations are applied to the observable on the science target, including those for detection biases as well as others associated with phasing errors, etc. Interleaved with observations of the science target are observations of calibrator stars, which would also be locally calibrated. From the calibrators, an estimate of the system leakage is determined and subtracted from the leakage on the science target. Similar to $\mathrm{V}^{2}$, the uncertainty in this calibrated residual is the most useful performance metric. A complete observation results from repeated observations of the science and calibrator stars. Incorporating other specific details of the observing scenario, $\sim 0.2 \%$ calibrated leakage allows detection of a 30 solar system equivalent zodiacal dust signature around a Sun-like star.

For $\mathrm{V}^{2}$ observations, a normalized visibility is determined by normalizing the coherent flux by the (squared) incoherent flux. Because of the difficulty of accurate 10 um photometry, we take a different approach with the nuller data, and include as part of the observing sequence measurements of the cross combiner signal with the nullers offset by $\pi$ to the fringe peak. The ratio of the coherent signal in the null and peak parts of the sequence is the normalized leakage. More detail on the observing strategy is provided in Ref. 11.

\subsection{Micro-Sequence}

While the four-aperture architecture addresses the detection of the zodiacal signal in the presence of both a bright star and a bright thermal background, the other "standard" interferometer issues remain, especially phase stabilization. While the star is bright relative to the zodi, its interferometric SNR on atmospheric time scales will generally be low, and the 10 um light cannot be relied upon for high bandwidth OPD control. In particular, in a single mode the leakage for a four- 
aperture nuller is proportional to the sum of the correlated phase $(\phi)$ and e-field amplitude (a) errors of the two nullers as

$$
\langle\text { Leakage }\rangle=\frac{1}{4}\left\langle\phi_{1} \phi_{2}+a_{1} a_{2}\right\rangle \text {. }
$$

For raw leakage of better than $1 \%$ at $10 \mathrm{um}$, the correlated phase errors must be controlled to $<300 \mathrm{~nm}$ rms; this is approximately how much the uncontrolled differential atmospheric OPD changes in $10 \mathrm{~ms}$. This is also a total rms requirement - not a windowed requirement as usually applies for $\mathrm{V}^{2}$ observations - and hence high frequency atmospheric fluctuations as well as low frequency fluctuations and drifts must be tracked. For our system, we use 2 um phase measurements to track the high frequency fluctuations, and lower bandwidth tracking at $10 \mathrm{um}$ to maintain the proper zero point. To accomplish this, we have implemented a time-multiplexed observing sequence built around a 4step micro-sequence as illustrated in Figure 2. A particular advantage of this implementation is that the detector and its spatial filter are exactly common mode in all sequence steps:

- Step 1: Track Nuller 1 using a standard ABCD algorithm ${ }^{16}$

- Step 2: Measure and track the coherent cross combiner signal with both nullers at Peak

- Step 3: Track Nuller 2

- Step 4: Measure the coherent cross combiner signal with both nullers at null

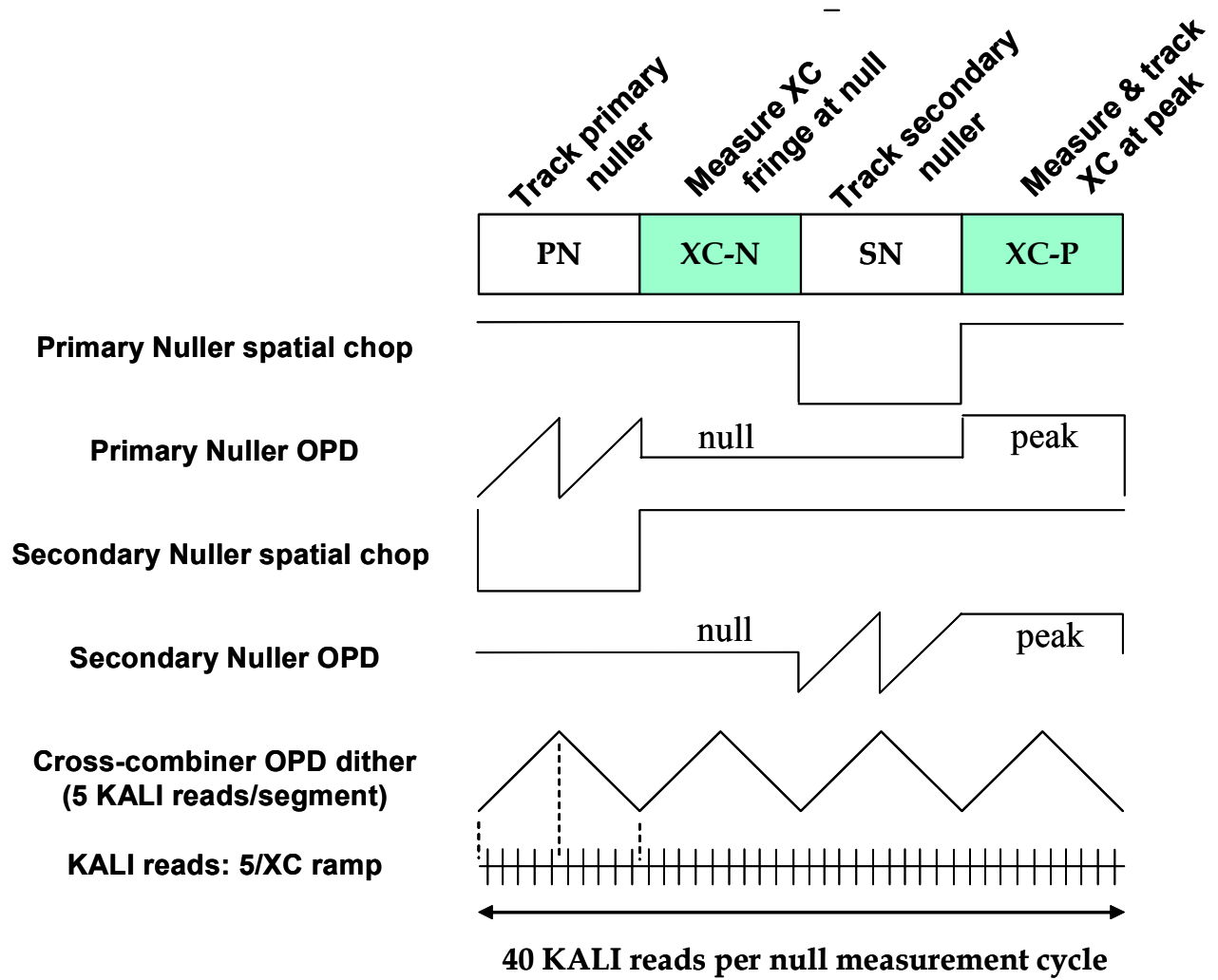

Figure 2. Nuller micro-sequence.

The micro-sequence takes $200 \mathrm{~ms}$, and adapts for fainter signals by changing the length of the underlying phasor averaging filters which filter the data used in the ABCD demodulation; clearly, lower control bandwidths are achieved with longer filters. Note that during step 2, we use the high SNR during the Peak-Peak measurement to track on the cross fringe: the stability requirements on the cross fringe are much looser than on the nuller fringe, as cross-fringe coherence loss drops out in the normalized leakage signal. Note that during steps 1 and 3, local chopper mirrors are used to off-point the light from Nullers 2 and 1, respectively; a standard phasor calibration step accommodates residual background fluctuations due to imperfect chopper motion. 
The nuller micro-sequence is almost entirely separate of the underlying high-bandwidth phasing system; the only communication between these system are "hold" signals if the phasing system drops out of lock or loses signal. The phasing system works transparently (to the nuller) to control high frequency pathlength fluctuations.

\subsection{Phase stabilization}

As described in detail elsewhere, ${ }^{5}$ and illustrated in Figure 3, star light is split between 2 and 10 um prior to the eight interferometer delay lines, and a pair of delay lines is used by each of the two nullers and each of the two 2 um fringe trackers ${ }^{17}$ for OPD control. The 2 um phasing system employs a similar hierarchical control approach as does the nuller. The highest frequency fluctuations are monitored by a series of laser interferometers and accelerometers. These are applied as targets to the optical delay lines, and the high frequency quieting they provide is transparent to the 2 um fringe trackers which track the mid and low frequency phase fluctuations attributable to atmospheric seeing and residual vibrations.

The fringe-tracker's phase measurements are used to provide standard feedback control to its local delay lines, and feedforward ${ }^{18}$ control to the delay lines associated with the nuller. The control distinction is significant: the fringetracker's delay lines are inside of the 2 um control loop, and thus standard servo stability criteria apply, limiting the amount of correction possible. However, the 10-um nuller's delay lines are not seen by the fringe tracker - we rely upon the laser metrology for accurate command following - so stability criteria do not apply and more of the error signal can be applied. Thus we add feedfoward of the 2 um servo error to the nuller delay line command, resulting in significantly improved error rejection, which is now limited primarily by sensor latency at high frequencies. The fringe tracker implements an overlapped ZABCD algorithm, and, with a $5 \mathrm{~ms}$ frame time and $1 \mathrm{~ms}$ updates, we achieve $\sim 4 \mathrm{~ms}$ total control latency including detection, processing, transport, and actuation. A lead filter in the feedforward controller further reduces the effective mid-frequency latency at the expense of some high frequency noise (largely uncorrelated; only correlated phase noise degrades the null). 


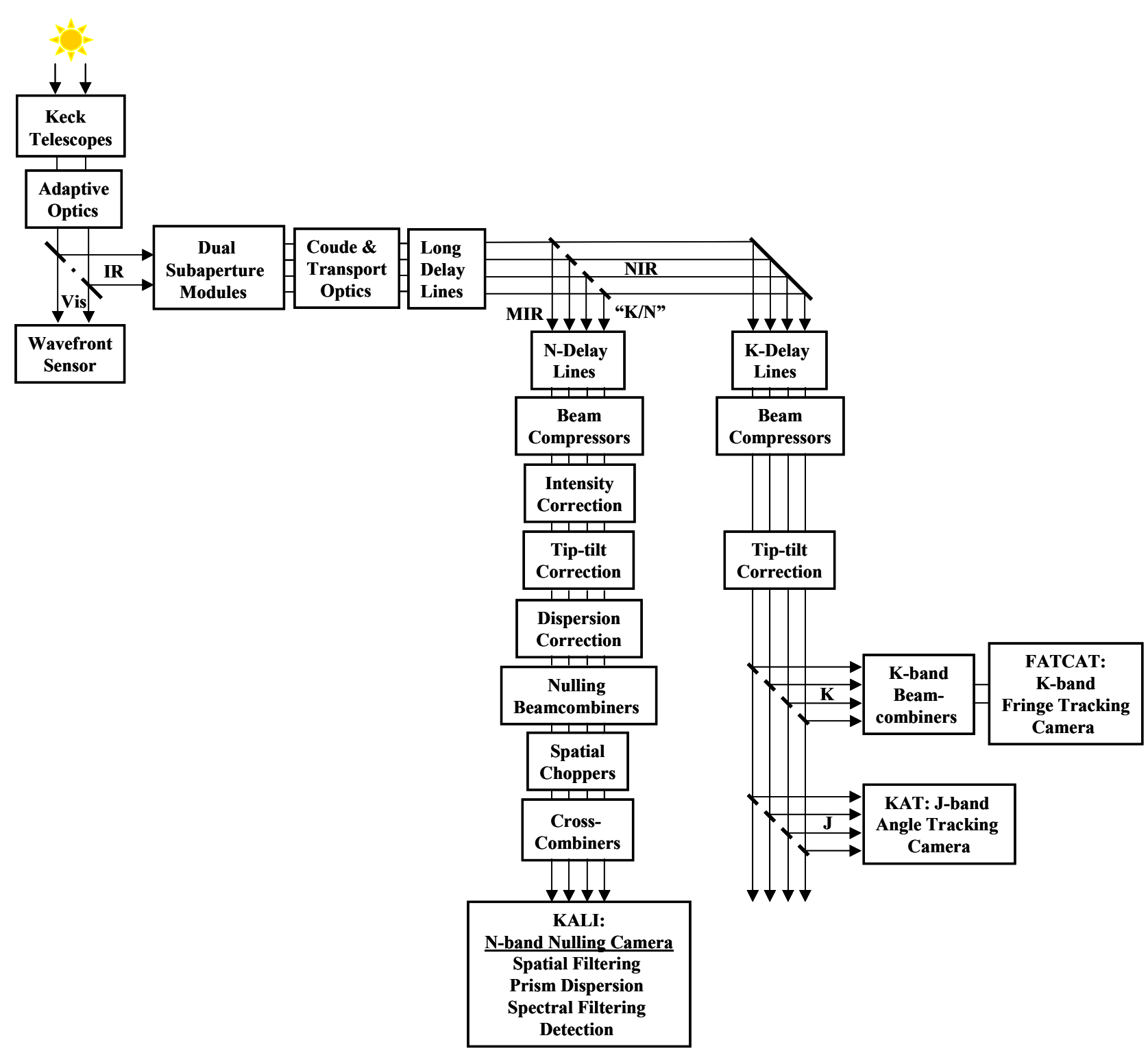

Figure 3. Block diagram of the nuller beamline.

As mentioned above, the local delay line stabilization is transparent to both the fringe tracker and the nuller. The delay lines servo to targets consisting of

- local FDL metrology: used for closed-loop control of the FDL servo

- internal (dc-coupled) differential metrology of the non-common parts of the fringe tracker and nuller optical paths

- external (ac-coupled) metrology of the common mode parts of the fringe tracker and nuller optical paths

- feedforward from the telescope accelerometers of piston beyond the final metrology fiducial

\subsection{Tilt stabilization}

High bandwidth tilt of the stellar beams is sensed at J- or H-band with an angle-tracking camera ${ }^{19}$ adjacent to the 2 um fringe tracker. The tip-tilt actuators include fast tip/tilt mirrors in front of both the nullers and fringe trackers. In this case, the mirrors seen by the nuller, which are outside of the J-band servo loop, are slaved to mirrors seen by the fringe 
(and angle) trackers. However, to minimize non-common errors, the low frequency tip/tilt commands are applied to a common mirror before the $10 \mathrm{um} / 2 \mathrm{um}$ split. This dual-actuator approach minimizes the signal amplitude on the noncommon mirrors to minimize errors caused by actuator non-linearity or gain matching. The tip/tilt mirrors include an optical encoder (using a retroreflected laser and a position sensitive detector) which can be employed if better matching is required.

The tip/tilt zero points are set separately for the fringe tracker and the nuller. For the fringe tracker, the pointing is optimized local to its beam combiner for optimal centration on the 2 um fibers, either using the raster capability within the fringe tracker, or manually to directly optimize the coupled flux.

For the nuller, pointing optimization requires explicit observation of a pointing star. The source is chopped using a left/center/right/center waveform (at two orientations) implemented within the AO system and synchronized with the angle tracker. The pointing star is chosen to be bright at $10 \mathrm{um}$ to yield both a high SNR and to avoid biases due to beamwalk attributable to chopping further downstream than is typical. A mirror local to the nuller beamtrain is adjusted to center the light on the nuller pinhole by symmetrizing the chopped intensity waveform.

\subsection{Group delay control}

The nuller observes over a greater than $20 \%$ bandpass, and control of 10 um group delay is required to achieve an achromatic null. A hierarchical control approach is taken here, too. The low frequency group delay control is accomplished directly by the nuller group delay servo, which controls in closed-loop an atmospheric dispersion compensator (ADC). However, the group delay is stabilized with respect to mid-frequency fluctuations by a feedforward signal from the fringe tracker which directly commands the ADC. Atmospheric water vapor fluctuations are the dominant contributor to nuller group delay fluctuations. However, these fluctuations can be estimated from the 2 um phase and group delay and a suitable dispersion model. ${ }^{20,21}$ This is implemented in the fringe tracker, generating an open-loop ADC command. In addition, the initial ADC zero point - the quasistatic dispersion due to water vapor within the interferometer beam paths - is estimated based on delay line position and the output of environmental sensors. Under conditions of moderate water vapor seeing, the feedforward from the fringe tracker significantly reduces the residual group delay fluctuations seen by the nuller, which for SNR reasons can only achieve a fairly low control bandwidth, keeping the nuller dispersion tracker within its dynamic range.

Water vapor fluctuations also contribute to differences between the 2 and 10 um (narrow-band) phases, leading to a residual error in the feedforward command from the fringe tracker to the nuller. This is also estimated from the 2 um phase and group delay, and is available for use on the faintest stars where the nuller control bandwidth is not adequate to reject the residual.

\subsection{Other aspects of the observing sequence}

As noted above, feedforward control can be used when the actuator is not observed by the sensor, and this is also taken advantage of in the sequence described in Figure 2. In particular, with respect to Nuller 1, while feedback control is implemented during step 1 when the nuller is being controlled closed-loop, the command switches to partial feedforward control during steps 2 and 4 to minimize the residual phasing error.

In addition, to accommodate residual instrumental chromatic terms, the nuller includes two slow loops to track a) the difference between the white light phase and the narrow band phase at a selected wavelength, and $b$ ) the difference between the fringe phase on one camera port versus the phase estimated from the sum of the phasors from both ports.

Finally, the determination of the phase track point used in steps 1 and 3 of the sequence bears discussion. We determine the offset using a "null phase offset" test whereby we measure the null leakage attributable to, say, Nuller 1, while we vary the phase track point on Nuller 2. From Eq. 1, it can be seen that the slope of the leakage with respect to $\phi_{2}$ gives the offset, $\phi_{1}$, of Nuller 2. This information is then used to update the servo track point. These measurements can be made in both internal (FTS) configuration and on-sky, with better null results obtained with the latter approach. We are also in the process of incorporating additional calibrations into our sequence to provide improved local calibration. 


\section{BEAMLINE UPDATE}

Other references $(1,5,6)$ provide considerable detail on the interferometer beamline. The following narrative discusses those elements which are different from the original $\mathrm{V}^{2}$ implementation.

\subsection{Telescope and adaptive optics}

The differences for nulling include the inclusion of neutral density filters for observations of the bright pointing reference stars, as well as replacement of the high-incidence-angle image-rotator coatings with bare aluminum to minimize polarization effects. (While the beamtrain maintains almost complete symmetry, low polarization coatings minimize second-order errors due to coating differences).

\subsection{Dual-star module and coude}

For nulling mode, a half-mirror is used to implement a left-right split of the collimated output from the AO system to provide two beams from each telescope. One beam is directed with the primary coude train to the base of the telescope; the other beam is directed with an active, secondary coude system. As the second coude system's optics are required to be slightly displaced from the telescope azimuth axis, two of the coude mirrors are tracked to an azimuth-dependent model to control pupil and angle. Secondary pointing errors are sensed by the angle tracker and ultimately continuously offloaded to one of the coude tracking mirrors, similar to the way primary pointing errors are offloaded to the AO system (strictly, the secondary offload is the difference between the secondary error and primary offload). We rely on the secondary coude model for pupil control, although we incorporate primary and secondary pupil checks in our observing sequence.

One of the fold mirrors for each of the left and right beams is a visible-transmitting dichroic, behind which is a metrology retroreflector. All of the fold mirrors at significant incident angles are bare gold to minimize polarizationdependent phase shifts (and polarization-dependent amplitude effects that can occur with overcoats at steep incidence angles).

\subsection{Beam transport and coarse delay}

At the base of the telescope, the primary and secondary beamtrains converge, and rectangular mirrors (one beam high; one beam low) direct light into the lab and through the long delay lines. As with the coude train, all 45-degree reflections are bare gold. The primary and secondary beamtrains diverge prior to the fast delay lines: half height mirrors direct the primary beams to one set of four delay lines, and the secondaries to the second set of four. These half height mirrors implement the common-mode tilt correction described above.

The primary and secondary beams are now split spectrally using beamsplitters, with $\mathrm{N}$ band light for the primary and secondary each going to pair of delay lines; and similarly for K (and J) band light.

\subsection{External metrology}

Prior to the spectral split are the four external metrology launchers (and associated source plate and electronics), each of which provides an ac-coupled delay line target to a pair of delay lines. These are mounted just off-axis of the stellar beam, and measure the common-mode $(\mathrm{K}$ and $\mathrm{N})$ pathlengths to the retroreflectors on the dual-star module.

\subsection{Delay lines}

The eight delay lines are all identical; two of these are used for visibility observations, with the rest used for nuller observations. Recent changes include control updates for better command following and changes to the fringe-tracker feedforward interface for reduced latency. An additional change is the inclusion of a target based on encoder read-back of the nuller ADC position. Through a glass dispersion model (and the metrology state), the delay line compensates for phase changes introduced by ADC motion, orthogonalizing the group-delay actuation for simplified phase control.

For the primary-side nuller beams, an OPD jog has been added to the beam on exit from the delay lines and subsequent beam compressors to ensure that cross fringes are obtained with close to the same FDL locations for both primary and 
secondary, maximizing our sky coverage. After the primary jog, the primary and secondary beams are directed into the nuller breadboard with gold-coated tip/tilt mirrors, with identical actuators to the (silver) tip/tilt mirrors for the K-band system.

Measuring through the delay lines are a set of 4 differential metrology systems starting from each of the two fringe trackers and each of the two Nullers. To minimize systematic errors, these utilize the starlight beamsplitters to split a dual-polarization beam between the two arms of each combiner. Polarizers at corner cubes located just below the Nband/K-band dichroics select the polarization for each arm. Before commencement of the nuller micro-sequence, the metrology systems are enabled to provide a dc-coupled target to the delay lines to control non-common errors.

\subsection{Fringe tracker}

There are now two identical fringe trackers, fed via single-mode fibers to 4 sub-arrays on the HAWAII array in the FATCAT dewar. ${ }^{17}$ Most of the recent fringe tracker changes are associated with the feedforward and water vapor systems, described above. However, in addition, we have changed the unwrapping strategy for nulling due to the nuller performance's dependence on a total rms vs. a windowed rms metric, and to simplify some other processing. For nulling mode the servo now tracks to zero phase delay rather than zero group delay, and uses a discrete unwrapper; for $\mathrm{V}^{2}$ mode, we continue to track to zero group delay using a continuous unwrapper.

\subsection{Nuller hardware}

The nuller back-end optics ${ }^{6}$ and camera ${ }^{7}$ have undergone only one significant change from previous descriptions, which is the incorporation of the spatial chopping mirrors for aid in implementation of the phasing steps in the micro-sequence. These are custom high-bandwidth one-axis angular choppers driven with a tailored waveform to minimize settling time. Smaller changes include improvements to the stimulus injection, and the encoding of the ADC noted above. Most of the other changes are in the real-time system and have been described above.

\subsection{Angle tracker}

The interferometer angle $\operatorname{tracker}^{19}$ now multiplexes four images onto its array, rather than two, but is otherwise not significantly changed. On recent update is a dewar internal alignment moving the optical axis closer to the array origin allowing a more efficient, and faster $(80 \mathrm{~Hz})$, clock pattern.

\section{CURRENT RESULTS}

Initial null results from 2005 were shown in Reference 5. Some preliminary results ${ }^{11}$ from the April 2006 nuller run are presented in Figure 4, which shows the calibrated leakage on a science star after calibration using a reference star, following the discussion in Section 2.1, with respect to the theoretical prediction. These data were obtained in "medium" mode using 3 pairs of observations of the science and calibrator stars. The integration times for these data are 10X longer than for the previously reported results (the coherent phasor filters for phase estimation are $2 \mathrm{~s}$ (clock time) long in gated mode). These recent observations incorporate all of the improvements noted above, including cophasing improvements, on-sky track-point updates, frequent shear adjustments, as well as a (real-time) track-point offset to optimize the null on one port.

\section{FUTURE PLANS}

At this point, all of the planned subsystems of the nuller are implemented, although several enhancements (such as use of the last two KALI ports, new K-band/N-band beamsplitters) remain. On sky testing has been invaluable in obtaining good performance, and all of the subsystems have benefited from this experience. While the basic observing sequence and observation scenario have been fairly stable over the past few runs, smaller changes are continuing to be made to improve performance and improve observing efficiency. These will continue over the next several runs as we move toward formal commissioning of the nuller mode in 2007. The nuller is currently available to the Keck community on a shared-risk basis: see http://msc.caltech.edu/software/KISupport.html for more details. 


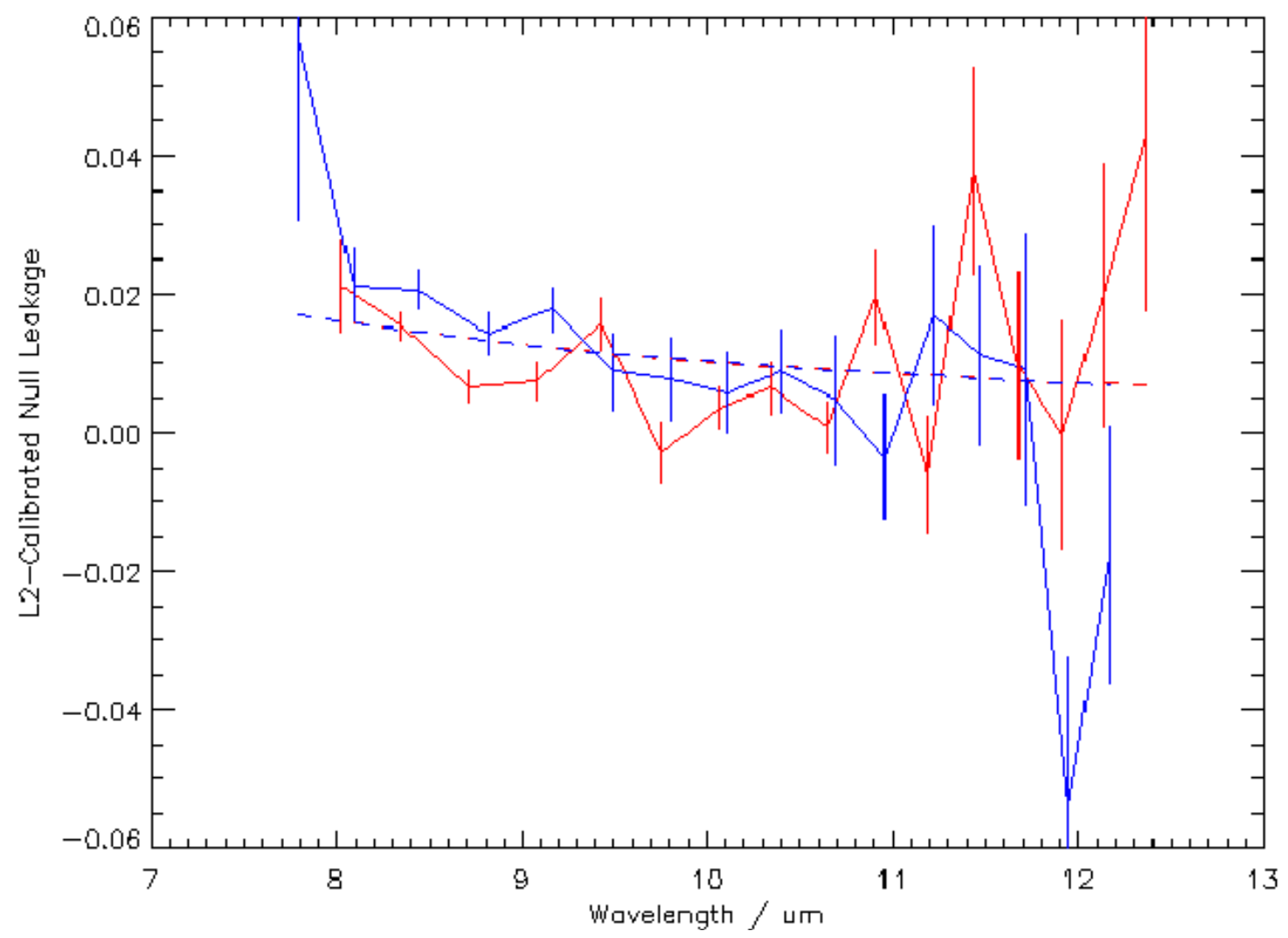

Figure 4. Preliminary calibrated null depth vs. wavelength from April 2006. The solid curves are null leakage vs. wavelength on the two nuller ports, and the dotted curve is the theoretical prediction.

\section{ACKNOWLEDGEMENTS}

The Keck Interferometer is funded by the National Aeronautics and Space Administration (NASA). Observations presented were obtained at the W. M. Keck Observatory, which is operated as a scientific partnership among the California Institute of Technology, the University of California, and NASA. The Observatory was made possible by the generous financial support of the W. M. Keck Foundation. Part of this work was performed at the Jet Propulsion Laboratory, California Institute of Technology, and at the Michelson Science Center, California Institute of Technology, under contract with NASA.

\section{REFERENCES}

\footnotetext{
${ }^{1}$ M. M. Colavita, P. L. Wizinowich, \& R. L. Akeson, 2004, "Keck Interferometer status and plans,” Proc. SPIE, 5491, 454.

${ }^{2}$ http://planetquest.jpl.nasa.gov/Keck

${ }^{3}$ P. L. Wizinowich, R. L. Akeson, et al., 2004, "Visibility science operations with the Keck Interferometer," Proc. SPIE, $5491,1678$.

${ }^{4}$ R. L. Akeson 2004, “Keck Interferometer science: present and future,” Proc. SPIE, 5491, 28.
} 
${ }^{5}$ E. Serabyn et al., 2005, “The Keck Interferometer nuller: configuration, measurement approach, and first results," Proc. SPIE, 5905, 59050T.

${ }^{6}$ E. Serabyn et al., 2004, “The Keck Interferometer nuller: system architecture and laboratory performance," Proc. SPIE, $5491,806$.

${ }^{7}$ M. J. Creech-Eakman, J. D. Moore, D. L. Palmer, \& E. Serabyn, 2003, "KALI camera: mid-infrared camera for the Keck Interferometer nuller," Proc. SPIE, 4841, 330.

${ }^{8}$ S. L. Crawford, et al. 2005, "Final laboratory integration and test of the Keck Interferometer nuller," Proc. SPIE, 5905, 59050U.

${ }^{9}$ A. J. Booth, M. M. Colavita, J. I. Garcia, \& C. D. Koresko, 2006, "The control system of the Keck Interferometer nuller," Proc. SPIE, 6268 (this conference).

${ }^{10}$ J. I. Garcia, M. M. Colavita, \& A. J. Booth, 2006, "Real-time control system for the Keck Interferometer nuller: methods and maintenance," Proc. SPIE, 6274 (this meeting).

${ }^{11}$ C. D. Koresko, M. M. Colavita, E. Serabyn, A. J. Booth, \& J. I. Garcia, 2006, "Measuring extended structure in stars using the Keck Interferometer nuller,” Proc. SPIE, 6268 (this conference).

${ }^{12}$ C. D. Koresko, M. M. Colavita, E. Serabyn, A. J. Booth, \& J. I. Garcia, 2006, "Water vapor measurement and compensation in the near- and mid-infrared with the Keck Interferometer nuller," Proc. SPIE, 6268 (this conference).

${ }^{13}$ E. Serabyn, 2006, "Science observations with the Keck Interferometer nuller," Proc. SPIE, 6268, (this conference).

${ }^{14}$ P. L. Wizinowich, M. M. Colavita, R. L. Akeson, 2006, "Recent progress at the Keck Interferometer," Proc. SPIE, 6268 (this conference).

${ }^{15}$ R. Millan-Gabet, 2006, “Keck Interferometer V2 science,” Proc. SPIE, 6268 (this conference).

${ }^{16}$ M. M. Colavita, 1999, "Fringe visibility estimators for the Palomar Testbed Interferometer," PASP, 111, 111.

${ }^{17}$ G. Vasisht, A. J. Booth, M. M. Colavita, et al., 2003, "Performance and verification of the Keck Interferometer fringe detection and tracking system," Proc. SPIE, 4838, 824.

${ }^{18}$ B. F. Lane \& M. M. Colavita, 2003, "Phase-referenced stellar interferometry at the Palomar Testbed Interferometer," Astron. J., 125, 1623.

${ }^{19}$ S. L. Crawford, A. J. Booth, M. M. Colavita, \& E. Hovland, 2006, "Design and performance of the Keck Interferometer angle tracker," Proc. SPIE, 6268 (this conference).

${ }^{20}$ M. M. Colavita, M. R. Swain, R. L. Akeson, C. D. Koresko, \& R. J. Hill, 2004, "Effect of atmospheric water vapor on infrared interferometry," PASP, 116, 876-885.

${ }^{21}$ C. D. Koresko et al., 2003, "Longitudinal dispersion control for the Keck Interferometer nuller," Proc. SPIE, 4838, 625. 